\title{
Response of the fungus Pseudocercospora griseola f. mesoamericana to Tricyclazole
}

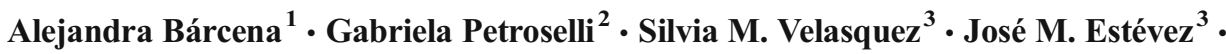 \\ Rosa Erra-Balsells ${ }^{2}$ • Pedro A. Balatti ${ }^{1,4,5}$ - Mario C. N. Saparrat ${ }^{1,4,6}$
}

Received: 4 May 2015 /Revised: 7 August 2015 / Accepted: 12 August 2015 / Published online: 25 August 2015

(C) German Mycological Society and Springer-Verlag Berlin Heidelberg 2015

\begin{abstract}
Pseudocercospora griseola, an anamorph of Mycosphaerella, causes Angular Leaf Spot (ALS). The mycelia and conidia from $P$. griseola are coloured due to the synthesis of 1,8 dihydroxynaphthalene (DHN)-melanin. The aim of this work was to identify in P. griseola $f$. mesoamericana isolate T4, a highly pigmented fungus, intermediary compounds as a result of the inhibition of melanin synthesis by tricyclazole, and to analyze at the structural level the localization of these dark pigments. The main metabolites were analyzed using ultraviolet matrix-assisted laser desorption-ionization mass spectrometry (UV-MALDI MS). Tricyclazole affected $P$. griseola $f$. mesoamericana in several different ways. The most evident effect was the reduction of melanin synthesis, and therefore diffusible shunt products were found and identified. Flaviolin was the main intermediate metabolite found in cultures supplemented with tricyclazole. This inhibitor, which affected pigmentation and the cell wall structure of mycelium, revealed macroscopically
\end{abstract}

Section Editor: Marc Stadler

Electronic supplementary material The online version of this article (doi:10.1007/s11557-015-1102-7) contains supplementary material, which is available to authorized users.

Mario C. N. Saparrat

masaparrat@yahoo.com.ar

1 Instituto de Fisiología Vegetal (INFIVE), Universidad Nacional de La Plata (UNLP)-CCT-La Plata-Consejo Nacional de Investigaciones Científicas y técnicas (CONICET), Diag. 113 y 61, CC 327, 1900 La Plata, Argentina

2 CIHIDECAR-CONICET, Departamento de Química Orgánica, Facultad de Ciencias Exactas y Naturales, Universidad de Buenos Aires, Pabellón II, 3 Ciudad Universitaria, 1428 Buenos Aires, Argentina by the reduction in growth, decreased the stratification and deposition of melanin in the hyphal wall. These results suggest a possible role of tricyclazole to control ALS.

Keywords Pseudocercospora griseolaf. mesoamericana . Phaseolus vulgaris · Melanin · Tricyclazole · UV-MALDI-TOF MS · Microscopy · FTIR

\section{Introduction}

Pseudocercospora griseola (Sacc.) Crous \& U. Braun, an anamorph of Mycosphaerella (Ascomycota), is the etiological agent of Angular Leaf Spot (ALS), a serious disease of common bean (Phaseolus vulgaris L.) around the world (Schwartz and Galvez 1980; Ddamulira et al. 2014) and the most important endemic disease in northwestern Argentina (20 to $50 \%$ of yield losses, Vizgarra et al. 2010). P. griseola coevolved with
3 Fundación Instituto Leloir, Av. Patricias Argentinas 435, C1405BWE Buenos Aires, Argentina

4 Cátedra de Microbiología Agrícola, Facultad de Ciencias Agrarias y Forestales, Universidad Nacional de La Plata, 60 y 119, 1900 La Plata, Argentina

5 Cátedra de Fitopatología-CIDEFI, Facultad de Ciencias Agrarias y Forestales, Universidad Nacional de La Plata, 60 y 119, CC31, 1900 La Plata, Argentina

6 Instituto de Botánica Spegazzini, Facultad de Ciencias Naturales y Museo, Universidad Nacional de La Plata, 53 \# 477, 1900 La Plata, Argentina 
its host, and much like the plant, the fungus has two major intraspecific groups, the formae $P$. griseola $f$. griseola (Andean group) and P. griseola $f$. mesoamericana (Mesoamerican group; Crous et al. 2006). Although both gene pools are pathogenic, P. griseola f. mesoamericana shows a broader range of virulence on bean varieties and a darker pigmentation and also tolerates higher temperatures, compared to those for P. griseola f. griseola (Crous et al. 2006).

Pseudocercospora griseola and other representatives of Mycosphaerellaceae synthesize dark pigments known as melanins. In contrast to other fungal species that need external precursors to synthesize this pigment (Franzen et al. 2008), $P$. griseola synthesizes melanins constitutively, which might be related to morphogenesis, pathogenesis and/or survival strategies of the fungus (Elliott 1995; Langfelder et al. 2003; Suryanarayanan et al. 2004). Recently, Saparrat et al. (2009) described that representatives of $P$. griseola from both gene pools synthesize 1,8-dihydroxynaphthalene (DHN) melanin. These same pigments have already been found in most Ascomycota, such as in Alternaria alternata (Carzaniga et al. 2002), Bipolaris oryzae (Moriwaki et al. 2004), Bipolaris sorokiniana (Kumar et al. 2015), Cladosporium cladosporioides (Llorente et al. 2012), Colletotrichum lagenarium, Magnaporthe grisea (Moriwaki et al. 2004), Pseudocercospora fijiensis (Beltrán-García et al. 2014) and Sporothrix schenckii (Romero-Martinez et al. 2000). However, information regarding the pathway of pigment synthesis as well as its localization at the ultrastructural level in P. griseola is lacking.

Strategies to manage ALS include cultural practices such as the use of resistance varieties of beans as well as chemical control (Stenglein et al. 2003; Paparu et al. 2014). The most effective fungicides to control $P$. griseola are dithiocarbamates and benzimidazols. While the former affect cytochrome P-450, the latter bind to tubulins, and this inhibits cellular division and therefore mycelial growth (Schwartz and Galvez 1980). Pigmentation of $P$. griseola hinders its control (Ddamulira et al. 2014). However, there is no information regarding the effect of these types of fungicides on melanin synthesis and/or their biological impact in this fungus. However, Llorente et al. (2012), while studying melanin synthesis on Cladosporium cladosporioides, found that Difenoconazole (Bogard $25 \%$, Syngenta Agro S.A., Argentina) and Clorotalonil (Daconil 72F, Syngenta Agro S.A., Argentina) generate a drastic reduction in fungal growth and conidiation. Concomitantly, cultures turned darker, most probably due to melanin deposition that seems to be a quencher of stress.

Tricyclazole (5- methyl-1,2,4-triazolo[3,4-b]benzothiazole; Fig. 1a) is a molecule that affects the DHN-melanin biosynthesis pathway through the inhibition of reductases that catalyze the transformation of $1,3,6,8$ - tetrahydroxynaphthalene $(1,3$, 6,8 -THN) to scytalone and of $1,3,8$ - trihydroxynaphthalene (1,
3, 8-THN) to vermelone (Bell and Wheeler 1986; Nobre and Ferreiro 2004). Saparrat et al. (2009) and Llorente et al. (2012) demonstrated that this fungicide reduced growth and melanization both on P. griseola and C. cladosporioides. Furthermore, this compound is used to control plant pathogens such as Magnaporthe grisea, Bipolaris oryzae, B. sorokiniana, Blumeria graminis, Colletotrichum lagenarium, C. lindemuthianum and Rhynchosporium secalis (Mares et al. 2004; Kunova et al. 2012; Kumar and Mukherjee 2013; Kumar et al. 2015).

The aim of this work was to evaluate the effect of tricyclazole on Pseudocercospora griseola $f$. mesoamericana growth, morphology and pigmentation when the fungus is grown in vitro. We studied melanin biosynthesis indirectly by identifying intermediary compounds as a result of the inhibitory activity of tricyclazole on reductases. Identifying biosynthetic intermediates provides an opportunity to investigate the role of the melanin in the causative agent of ALS and the potential use of tricyclazole to control it. Furthermore, we studied the localization of these dark pigments in fungal cells by Fourier Transform Infrared (FTIR) and microscopical analysis, methods that are diagnostic of melanin (Kayatz et al. 2001; Tavzes et al. 2009; Llorente et al. 2012).

\section{Materials and methods}

\section{Fungal material}

Isolate T4 of $P$. griseola representing the Mesoamerican (P. griseola f. mesoamericana) group was obtained from a typical leaf spot symptom (Stenglein and Balatti 2006). This fungal isolate was deposited in the Culture Collection of the Centro de Investigaciones de Fitopatología (CIDEFI), Facultad de Ciencias Agrarias y Forestales, Universidad Nacional de La Plata as CIDEFI 232. It was kept at $-20{ }^{\circ} \mathrm{C}$ on filter paper impregnated with a conidia-mycelium suspension and as a culture grown for 7 days on a potato dextrose agar (PDA) medium at $24 \pm 2{ }^{\circ} \mathrm{C}$ and maintained at $4{ }^{\circ} \mathrm{C}$. Inoculation was carried out by using a conidial suspension obtained according to Llorente et al. (2012), and unless specifically stated, cultures on PDA (control) and PDA supplemented with tricyclazole (Ultra Scientific Analytical Solutions, United States) were incubated in the dark at $24 \pm 2{ }^{\circ} \mathrm{C}$ for 21 days.

\section{Isolation and identification of melanin pathway intermediates by UV-MALDI MS}

Intermediate metabolites of the fungus that was grown on PDA (control) and PDA supplemented with tricyclazole were 
Fig. 1 Chemical structure of (a) tricyclazole, an inhibitor of 1,8DHN melanin synthesis, and (b) flaviolin, the shunt product generated by the inhibition of the DHN-melanin biosynthesis pathway in $P$. griseola $f$. mesoamericana by tricyclazole

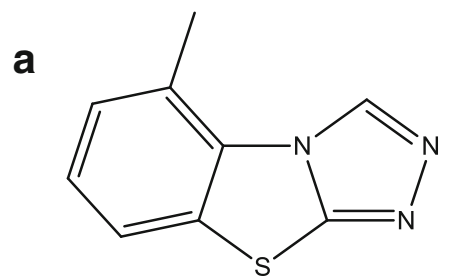

extracted with ethyl-acetate (Wheeler and Klich 1995). A representative culture growing on either PDA (control) or PDA supplemented with $100 \mathrm{ppm}$ of tricyclazole was treated with ethyl-acetate for 2 and $24 \mathrm{~h}$. On aliquots from the two ethylacetate extracts obtained from each culture type, three independent UV-MALDI analytic measurements were conducted. This procedure was repeated in a second experiment using the same replicate number. As a reference, we used a freshly prepared ethanolic solution of standard tricyclazole $\left(2 \mathrm{mg} \mathrm{ml}^{-1}\right)$ in a straightforward manner. Samples were analyzed by ultraviolet matrix assisted laser desorption-ionization mass spectrometry (UV-MALDI MS) performed on the Bruker Ultraflex Daltonics TOF/TOF mass spectrometer. In preliminary assays, several compounds ( $\alpha$-cyano-4-hydroxycinnamic acid, CHCA; sinapinic acid, SA; 2,5dihydroxybenzoic acid (gentisic acid), GA; 9H-[3,4b] piridoindole (norharmane), nHo) were evaluated as MALDI matrix showing similar broad signals for the molecular ions. Mass spectra were acquired in linear positive and negative ion modes and with the LIFT device in the MS/MS mode. External mass calibration was made using $\beta$ cyclodextrin (MW 1134) with nHo and GA as matrices in positive and negative ion mode. Sample solutions were spotted on a MTP 384 target plate polished steel from Bruker Daltonics. Matrix solutions were prepared by dissolving nHo or GA in water $\left(1 \mathrm{mg} \mathrm{ml}^{-1}\right)$. Dry droplet sample preparation (sandwich method) was used according to Nonami et al. (1997), loading $0.5 \mu \mathrm{l}$ of matrix solution, analyte solution and matrix solution successively after drying each layer at normal atmosphere and room temperature. The matrix to analyte ratio was $3: 1\left(\mathrm{v} \mathrm{v}^{-1}\right)$ and the matrix and analyte solution loading sequence was: 1) matrix, 2) analyte, 3) matrix, 4) matrix. Desorption/ Ionization was obtained by using the frequency-tripled $\mathrm{Nd}$ : YAG laser (355-nm). The laser power was adjusted to obtain high signal-to-noise ratio $(\mathrm{S} / \mathrm{N})$ while ensuring minimal fragmentation of the parent ions and each mass spectrum was generated by averaging 100 lasers pulses per spot. Spectra were obtained and analyzed with the programs FlexControl and FlexAnalysis, respectively. The resolution of the signals detected was estimated according to their Full Width at Half-Maximum (FWHM), which in most cases was approx. $0.8 \mathrm{~m} / \mathrm{z}$ unities.

\section{Fourier transform infrared (FTIR)}

Since FTIR has been used to identify several melanins in polymeric matrices by the presence of specific functional groups and chemical bonds in their structure (Tavzes et al. 2009; Tu et al. 2009; Llorente et al. 2012), the chemical composition of dry mycelium samples from three 21-day-old cultures grown on PDA (control) and PDA supplemented with tricyclazole at $100 \mathrm{ppm}$ were analyzed by means of the potassium bromide (KBr) method on a Bruker Fourier-transform IR spectrophotometer. The fungal material from both types of cultures was washed with water at $100{ }^{\circ} \mathrm{C}$ for $5 \mathrm{~min}$. Then, the resultant suspension was centrifuged at 5,000 $\mathrm{g}$ for $5 \mathrm{~min}$ and the pellet was dried in an oven at $40{ }^{\circ} \mathrm{C}$ overnight. Dry mycelium was embedded in infrared grade $\mathrm{KBr}$ disk (2 mg $20 \mathrm{mg}^{-1}$ ) and recorded in a Perkin-Elmer-Instruments spectrometer (scanning range: $400-4,000 \mathrm{~cm}^{-1}$, resolution: $4 \mathrm{~cm}^{-1}$, number of scans: 64), the data of which was processed by means of the software OMNIC (Thermo Nicolet, Madison, WI) according to Saparrat et al. (2010). This experiment was performed once with three replicates.

\section{Microscopical analysis}

Representative samples of mycelium taken from three 21-dayold cultures growing on PDA (control) and PDA supplemented with tricyclazole at $100 \mathrm{ppm}$ were observed with light (LM), transmission electron (TEM) and confocal (CM) microscopes. Each experiment was performed twice.

For LM, the samples were directly observed with an Olympus BX51 light microscope equipped with an Olympus E-330 digital camera.

Samples for TEM were fixed with $2 \%$ glutaraldehyde $2 \mathrm{~h}$ at $4{ }^{\circ} \mathrm{C}$ and then washed with phosphate buffer. They were post fixed $2 \mathrm{~h}$ at $4{ }^{\circ} \mathrm{C}$ with $1 \%$ osmium tetroxide, washed with phosphate buffer, dehydrated with ascending grades of ethanol. The procedure ended with samples inmersed in acetone. Then samples were embedded in Epoxy resin. Ultrathin sections of $60 \mathrm{~nm}$ were cut on a Reichart-J Super Nova Ultracut. Sections were placed on copper grids and stained with uranyl acetate $1 \%$ and lead citrate (Kogej et al. 2006). Samples were viewed on a JEOL JEM 1200 EX II TEM and micrographs were taken with a digital camera Erlangshen ES $1000 \mathrm{~W}$. 
Samples for in situ detection of melanin in cell walls were oxidized by exposing to $\mathrm{H}_{2} \mathrm{O}_{2} 6 \mathrm{M}$ and light for $1 \mathrm{~h}$ according to Kayatz et al. (2001), and seen by CM in an Olympus FV300 CM (Olympus Latin America) equipped with a 60 X 1.2 N.A. water immersion objective. Melanin fluorescence was detected using a $488 \mathrm{~nm}$ laser (excitation) and emission filters at $505-525 \mathrm{~nm}$. Since autofluorescence of vesicles was found at this range of wavelength when samples were not exposed to hydrogen peroxide and light, the amount of fluorescence signal on both cell wall and intracellular particles was processed and quantified according to Ricardi et al. (2012). Means of the treatments were contrasted by the Student's $t$ Test (at $p \leq$ 0.05 ) using the Statistix 8.0 software for Windows.

\section{Colony growth and pigmentation under a range of tricyclazole concentrations}

The PDA medium was amended with tricyclazole at a concentration of 12.5, 25, 100 and 200 ppm (van de Sande et al. 2007). Colony growth was estimated by determining colony diameter and color was measured by estimating darkness $(\mathrm{k})$ of the colonies. To do this, scanned images of colonies were analysed with a grey scale using Adobe ${ }^{\circledR}$ Photoshop ${ }^{\circledR}$ 8.0.1 (Griffith et al. 2007). The relationship between the percentage of growth inhibition and the concentration of the fungicide was estimated; this allowed us to calculate the concentration of fungicide that caused $50 \%$ of growth inhibition $\left(\mathrm{IC}_{50}\right.$; Llorente et al. 2012). This experiment was performed twice with three replicates at each concentration. Data were analyzed by means of a one-way analysis of variance (ANOVA) and means of the treatments were contrasted by the Tukey Test (at $p \leq 0.05$ ) using the Statistix 8.0 software for Windows.

\section{Results}

UV-MALDI-mass spectrometry analysis of metabolites from ethyl-acetate extracts of $P$. griseola $f$. mesoamericana determined the presence of DHN-melanin precursors or related metabolites. Figure 2 shows UV-MALDI mass spectra of the samples obtained from 21-day-old cultures of isolate T4 grown on PDA without (control) and with addition of tricyclazole $100 \mathrm{ppm}$ (Fig. 2a and b, respectively). In the presence of tricyclazole, a signal at $\mathrm{m} / \mathrm{z}=206.52$ was detected, compatible with the compound flaviolin (2,5,7-trihydroxy-1, 4-naphthoquinone; Fig. 1b, exact mass 206.02), which is an oxidation product of 1,3,6,8-THN. This was confirmed by MS/MS spectrum of the ion detected $\left[\mathrm{M}^{+}, \mathrm{C}_{10} \mathrm{H}_{6} \mathrm{O}_{5}\right]$ and its fragmentation pattern (Fig. S1 and Scheme S1). This signal at $\mathrm{m} / \mathrm{z} 206$ was absent in the control (Fig. 2a) and is not a matrix

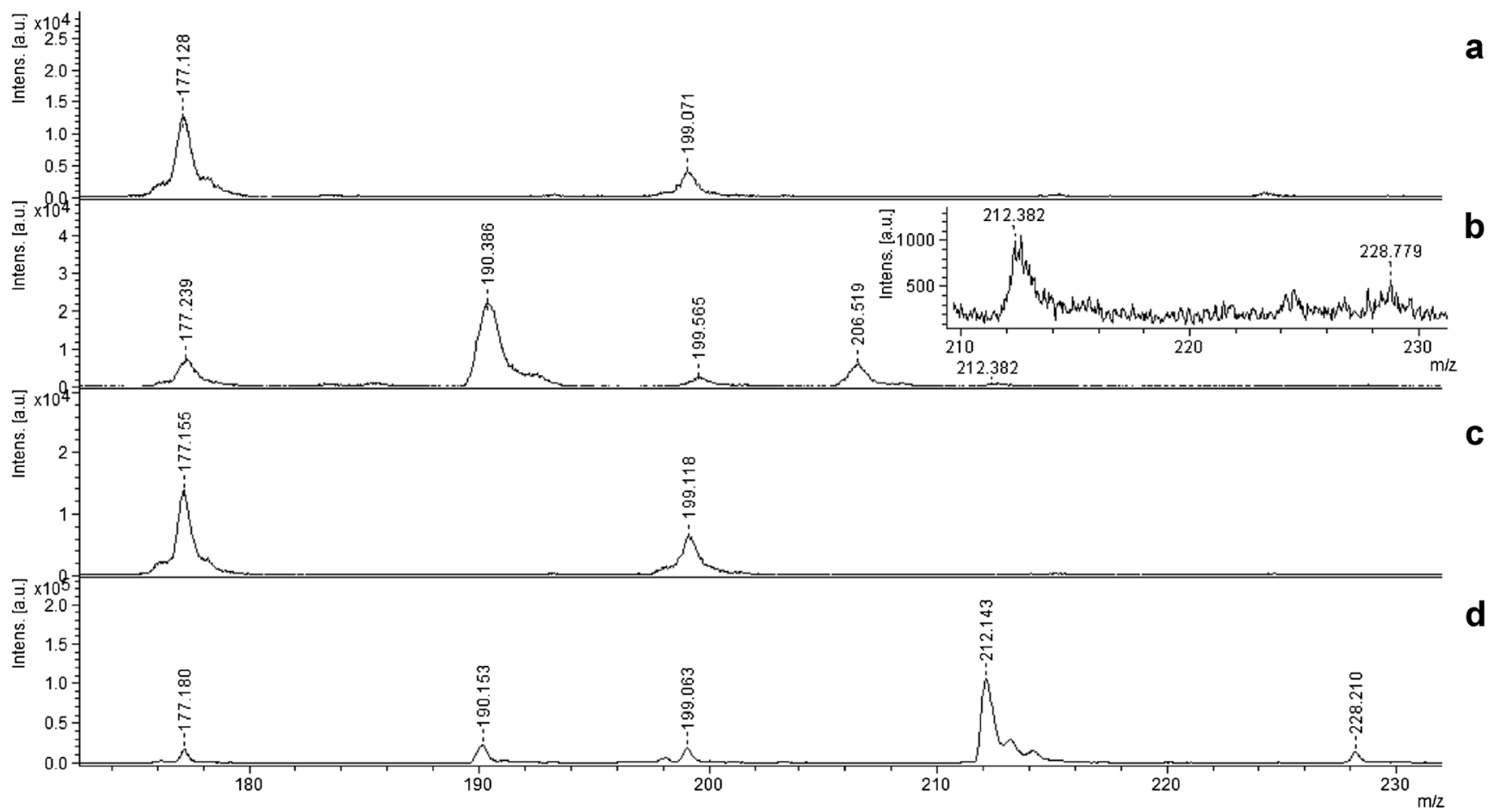

Fig. 2 Positive ion MALDI mass spectra of ethylacetate extract corresponding to 21-day-old cultures of T4 grown on PDA: a) control $24 \mathrm{~h}$; b) supplemented with $100 \mathrm{ppm}$ tricyclazole $24 \mathrm{~h}$, including an inset shows the zoom between $\mathrm{m} / \mathrm{z}$ region 210 and 230; c) gentisic acid

(matrix) and d) tricyclazole. Matrix: gentisic acid. Peaks assignment:

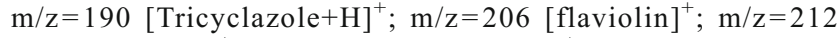
$[\text { Tricyclazole }+\mathrm{Na}]^{+} ; \mathrm{m} / \mathrm{z}=228[\text { Tricyclazole }+\mathrm{K}]^{+}$ 
cluster (Fig. 2c). Concomitantly with this, the mycelium and extracellular medium of tricyclazole-grown cultures developed a reddish coloration (Fig. 5b). Under such conditions, a signal at $\mathrm{m} / \mathrm{z}=190.39$ was detected (Fig. 2b). In order to determine if this signal corresponds to the intermediate 2hydroxy juglone (2-HJ; exact mass $=190.02)$ or to protonated tricyclazole (exact mass $=190.04$ ), the mass spectrum of tricyclazole was measured (Fig. 2d). As expected, the protonated $(\mathrm{m} / \mathrm{z}=190.15)$, sodiated $(\mathrm{m} / \mathrm{z}=212.14)$ and potassiated tricyclazole $(\mathrm{m} / \mathrm{z}=228.21)$ ions were detected. Additionally, UV-MALDI MS/MS mass spectra of the ion with $\mathrm{m} / \mathrm{z}=190$ observed in the tricyclazole spectrum and that observed at similar $\mathrm{m} / \mathrm{z}$ value in the spectrum of ethyl-acetate extract in the presence of inhibitor were obtained (Fig. 3). The fragmentation patterns of both MS/MS spectra obtained were similar, indicating that the later signal (Fig. 2b) corresponded to the tricyclazole moiety. Furthermore, symmetric Gaussian patterns in the signals detected on the UVMALDI MS spectra for flaviolin (Fig. 2b) and tricyclazole (Fig. 2b and d) showed relevant levels of intensity (approx. 6000 a.u.), which according to $\mathrm{m} / \mathrm{z}$ values, is accurate and therefore reveals the presence of a single molecule species.

The FTIR spectra of dry mycelia from control as well as $100 \mathrm{ppm}$ tricyclazole-treated cultures presented similar bands of absorption. They were characterized by peaks between $1670-1600 \mathrm{~cm}^{-1}$ that correspond to conjugated double bonds $\mathrm{C}=\mathrm{C}$ and $\mathrm{C}=\mathrm{O}$ in the aromatic ring as well as $\mathrm{C}=\mathrm{O}$ in secondary amines, diagnostic features of typical conjugated quinoid structures such as those of melanins (Fig. 4).

Hyphae and conidia of cultures supplemented or not supplemented with 100 ppm tricyclazole were analyzed microscopically (Fig. 5a and b). When control cultures were examined by LM, it was observed that fungal walls were melanized (Fig. 5c). In fungi grown with tricyclazole, cell walls were clearer (Fig. 5d). Further analysis with TEM showed that while the hyphal walls from mycelia developed on control PDA presented an organized inner electron-transparent layer and an outer electron-dense one, those from fungi grown with tricyclazole did not (Fig. 6). Furthermore, when mycelia were treated with $\mathrm{H}_{2} \mathrm{O}_{2}$ and light, and were then examined with $\mathrm{CM}$, a fluorescent melanin-type material was found in the cell wall of all cultures (Fig. 7a.2 and b.2). Additionally, when the fungal material was not treated with $\mathrm{H}_{2} \mathrm{O}_{2}$-light, no fluorescence in the hyphal wall was detected (Fig. 7a.1 and b.1), but lower signal was detected in cultures grown with tricyclazole (Fig. 7b.2), and an autofluorescent signal for intracellular vesicles was found both in the control cultures and in those treated with tricyclazole. Therefore, a higher cell wall/vesicles fluorescence relationship was found in control cultures treated with $\mathrm{H}_{2} \mathrm{O}_{2}$-light than in those from cultures supplemented with tricyclazole (Fig. 8).

Isolate T4 was grown on PDA medium and PDA supplemented with $12.5,25,100$ and 200 ppm tricyclazole, which affected growth and pigmentation (Fig. 9a and b respectively). This increase in concentration led to the development of

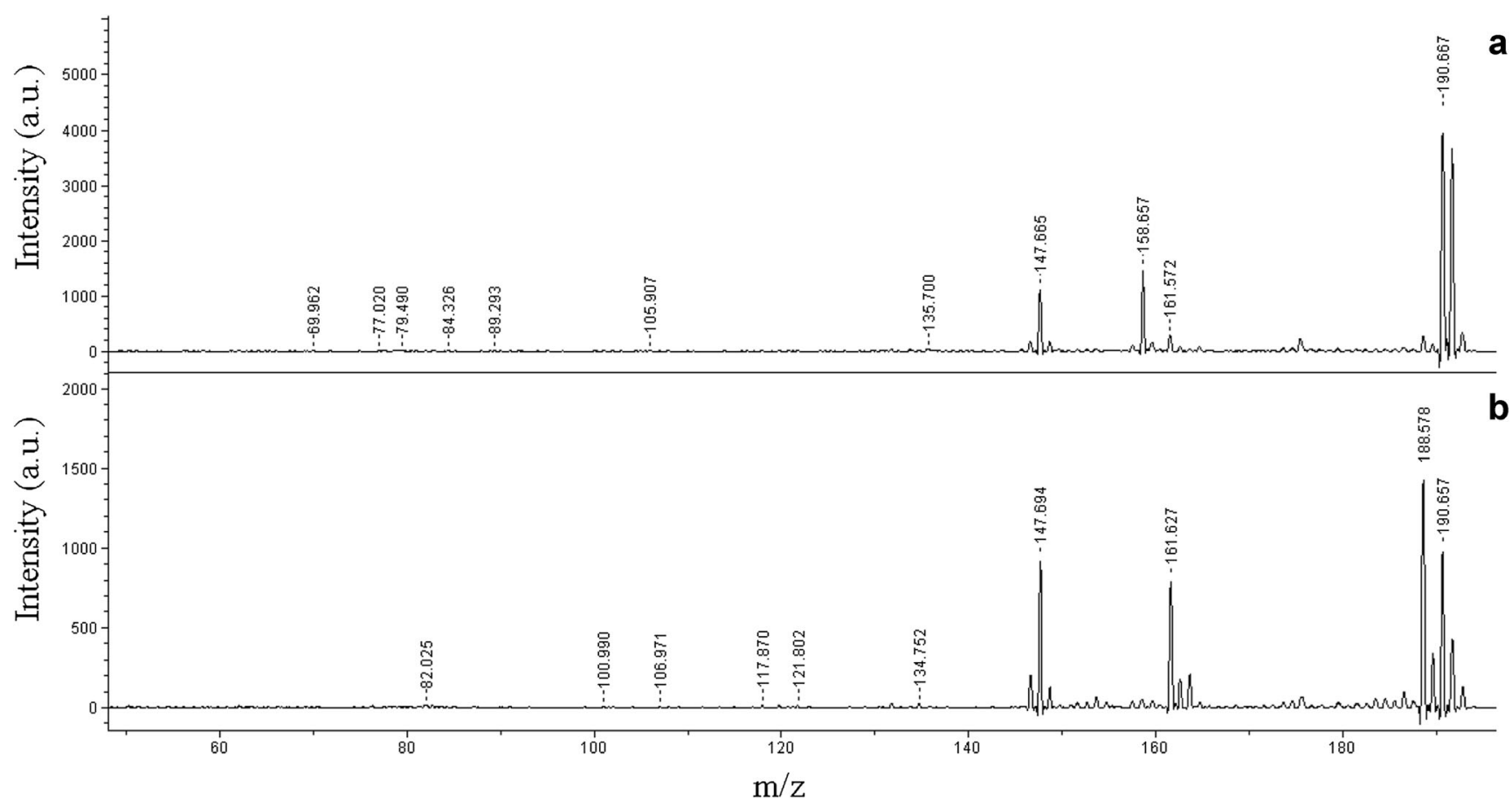

Fig. 3 Positive ion UV-MALDI MS/MS mass spectra of $[\mathrm{M}+\mathrm{H}]^{+}$ion with $\mathrm{m} / \mathrm{z} 190$ from a) ethylacetate extract corresponding to 21-day-old cultures of T4 grown on PDA supplemented with $100 \mathrm{ppm}$ tricyclazole $24 \mathrm{~h}$ and b) tricyclazole standard 

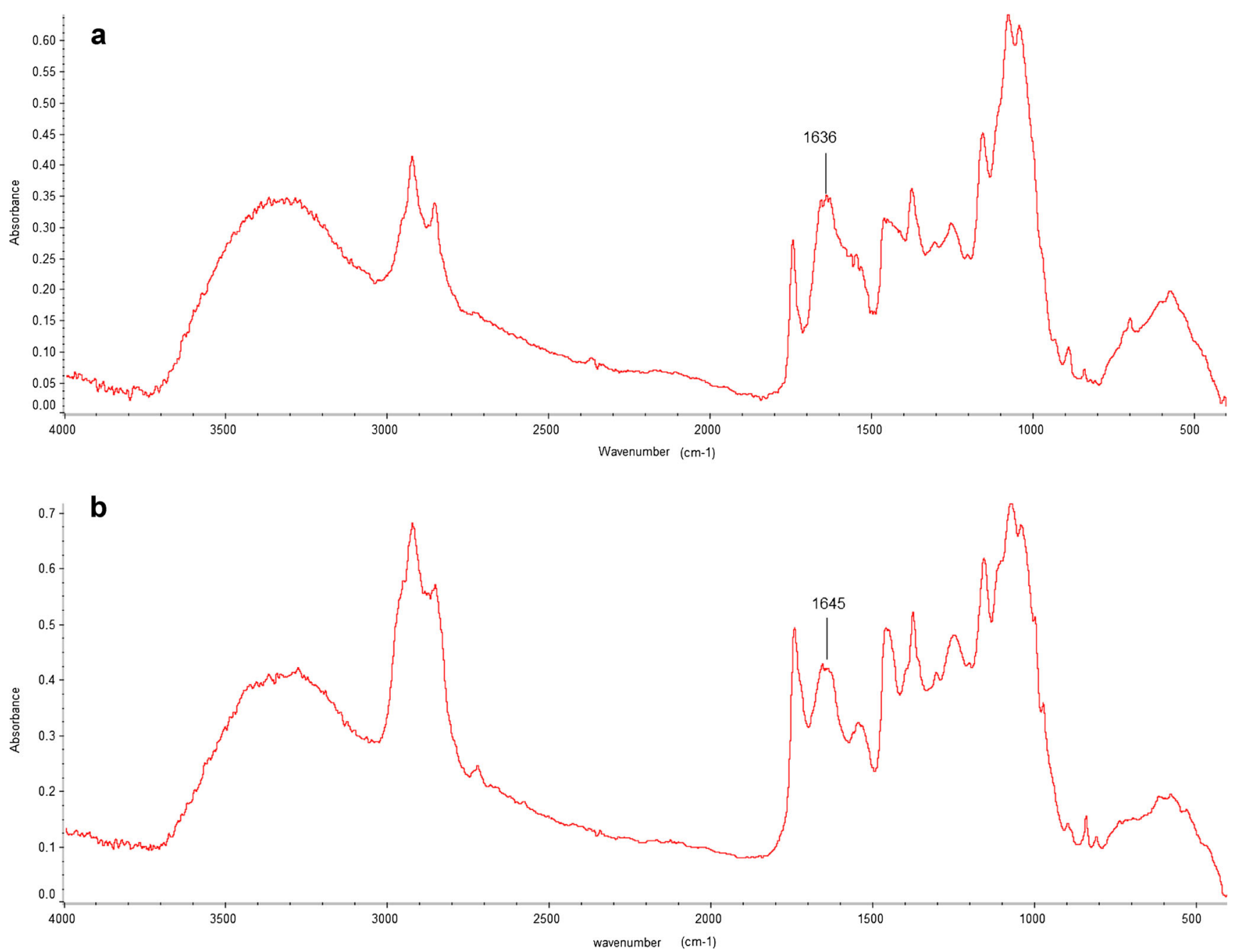

Fig. 4 FTIR spectrum of mycelium of P.griseola f. mesoamericana grown on PDA (basal medium) (a) and on PDA+100 ppm of tricylazole (b). This experiment was performed once with three repetitions

lighter colonies, which resulted in a reduction of the darkness "k" from 12.5 ppm (Fig. 9b). Though colony size was substantially reduced at $100 \mathrm{ppm}$ (Fig. 9a), the concentration of fungicide that caused $50 \%$ reduction of growth was $118.75 \mathrm{ppm}$.

\section{Discussion}

Melanins are widely distributed among dematiaceous fungi such as Mycosphaerellaceae (Hoss et al. 2000). These polymeric substances might have a biological role such as virulence and/or survival against environmental stress factors (Bell and Wheeler 1986; Cooke and Whipps 1993; Chaskes et al. 2008; Onofri et al. 2008; Singaravelan et al. 2008; Taborda et al. 2008). Saparrat et al. (2009) showed that Pseudocercopora griseola synthesizes DHN-melanins, a process that was inhibited by tricyclazole. Here, we confirmed that this inhibition leads to a reduction in fungal growth and pigmentation. While $12.5 \mathrm{ppm}$ of tricyclazole affected colony pigmentation, it did not affect fungal growth, at least at such concentration. Fernandez et al. (2013) also reported that melanization of Cenococcum geophilum was sensitive to tricyclazole, but it was mostly unrelated to fungal growth. A similar reduction in pigmentation was observed in several phytopathogenic fungi that produce DHN-melanin, like Alternaria solani, Botrytis cinerea, Magnaporthe grisea, Sclerotinia minor and Thielaviopsis basicola, as well as in the human pathogens Aspergillus terreus, Aspergillus tubingensis, Nannizzia cajetani and Trichophyton violaceum (Mares et al. 2004).

Regarding synthesis of other pigments, when isolate T4 was grown in the presence of tricyclazole, a reddish pigment diffused out of the mycelium. Our results suggest that tricyclazole reduced rather than blocked melanin synthesis since the FTIR analysis, such as reported by Tavzes et al. 
Fig. 5 Colony, mycelia and conidia of P.griseola $f$. mesoamericana grown on PDA in the absence $(\mathrm{a}+\mathrm{c})$ and presence of $100 \mathrm{ppm}$ of tricyclazole $(b+d)$. Scale $20 \mu \mathrm{m}$

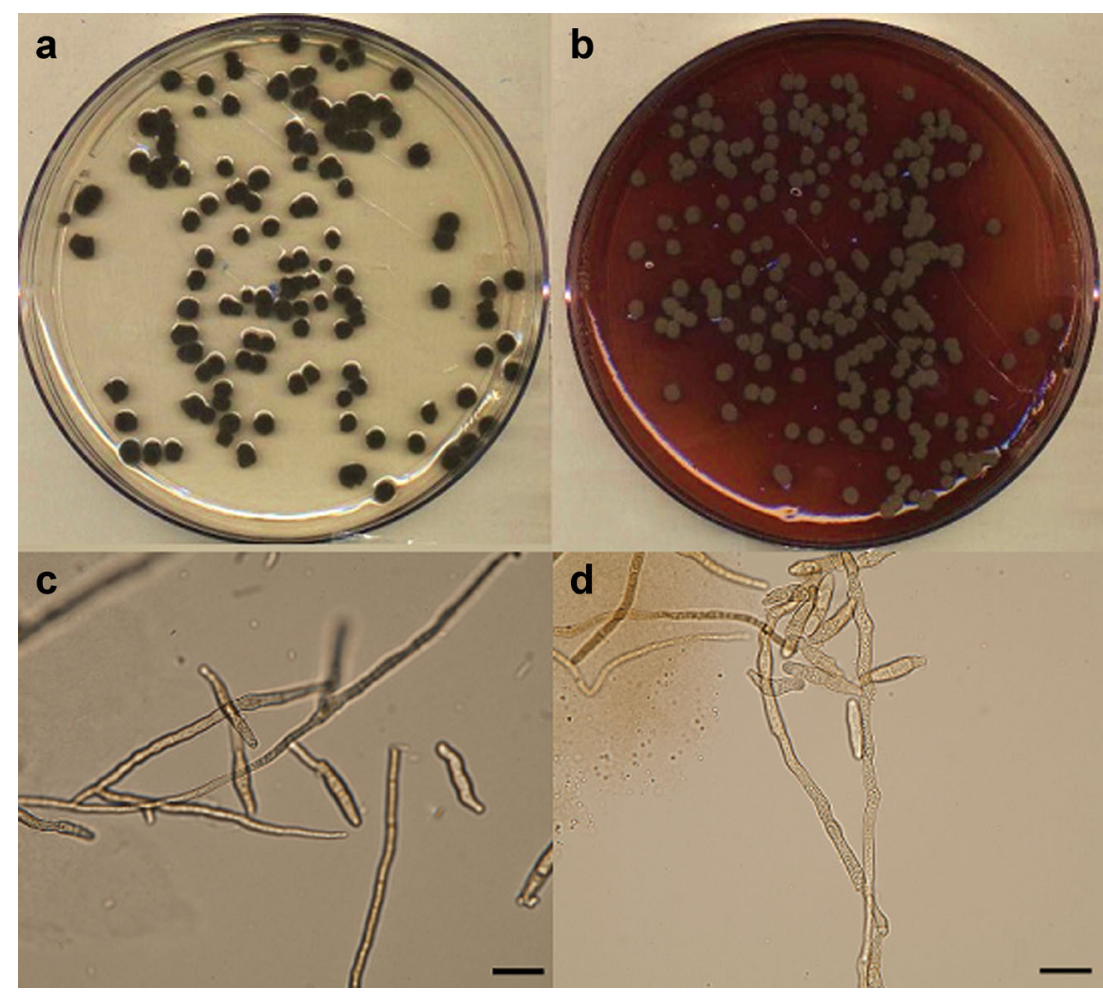

(2009), revealed the presence of melanin in hyphal walls whether the fungus was or was not grown in the presence of the inhibitor. Concomitantly with this, the reddish pigment was released. Based on all these evidences, Saparrat et al. (2009) hypothesized that the reddish pigments might be the soluble compounds released from fungal cultures grown in the presence of tricyclazole, which might be intermediate metabolites of melanin. Such a reduction in melanin synthesis, at least at certain concentrations of the inhibitor, affected mycelial growth; therefore, the melanin level in fungal wall may also be a determining factor in the organization of cell structure.

UV-MALDI MS is a key analytical tool to identify the chemical nature of compounds of low molecular mass, such as intermediate metabolites involved in melanin synthesis. In this work, we found that such an intermediate metabolite had a MS/MS spectrum and a retro Diels-Alder like fragmentation pattern typical of flaviolin (McLafferty and Turecek 1993), which was the main metabolite associated with mycelium of $P$. griseola $f$. mesoamericana grown on medium with tricyclazole $100 \mathrm{ppm}$, probably as a result of the autoxidation of 1, 3, 6, 8-THN. Flaviolin might be a shunt product from the inhibition of reductases by tricyclazole (Zeng et al. 2012), since isolate T4 synthesizes melanin through the DHNpathway (Saparrat et al. 2009).

Furthermore, no trace of 2-HJ, another diagnostic tool to identify DHN-melanin (Butler and Day 1998), was found. In cultures of Alternaria solani grown with high concentrations of tricyclazole, DHN-melanin was inhibited, 2-HJ was found and traces of flaviolin were detected (Lazarovits and Stoessl
Fig. 6 Hyphae of P.griseola $f$. mesoamericana isolate $\mathrm{T} 4$ grown on PDA (basal medium) (a) and on PDA+100 ppm of tricylazole (b). Lines indicate layers with different electron-density

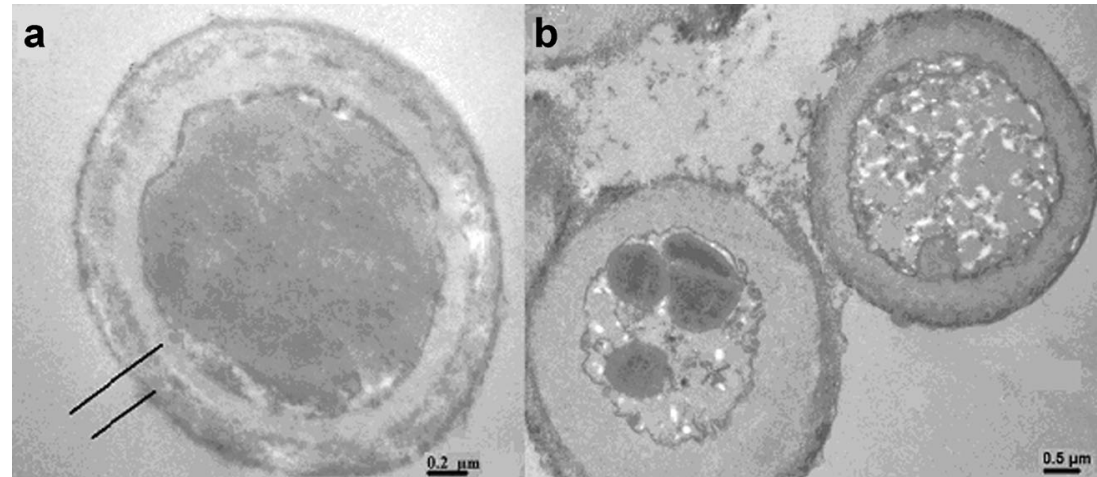



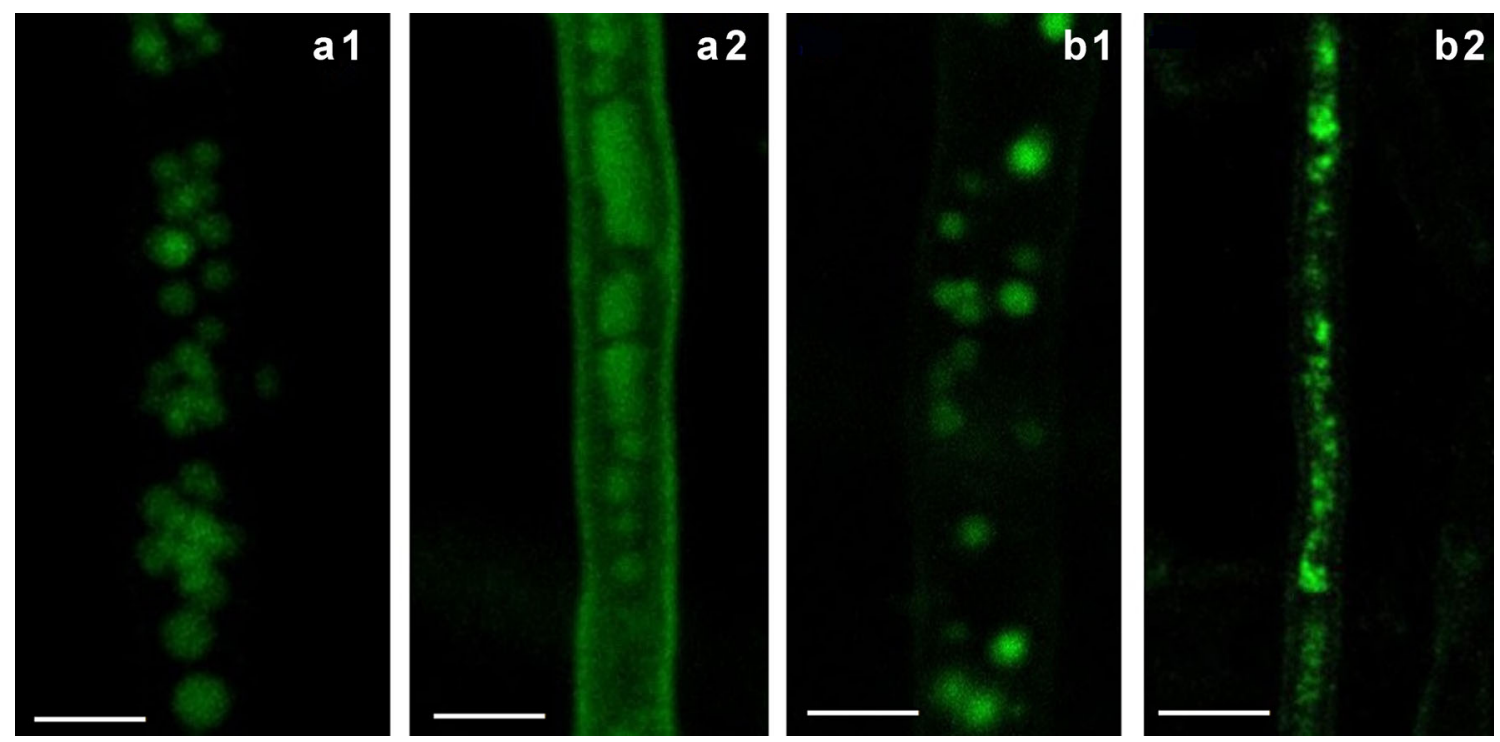

Fig. 7 Hyphae of P.griseola f. mesoamericana isolate T4 from cultures grown on PDA alone (a) and on PDA+100 ppm of tricylazole (b), either nontreated (1) or treated (2) with $\mathrm{H}_{2} \mathrm{O}_{2} 6 \mathrm{M}$ and light. Scale $5 \mu \mathrm{m}$

1988). In cultures of Verticillium dahliae, 1 to $20 \mathrm{ppm}$ of tricyclazole resulted in an increase of 2-HJ and a decrease in flaviolin level (Lazarovits and Stoessl 1988). According to Lazarovits and Stoessl (1988), the type of melanin shunt products in the presence of tricyclazole and their accumulation are dependent on the fungicide concentration and the fungus tested. Since we only extracted the metabolites from cultures grown at one concentration of tricyclazole, the absence of traces of 2-HJ might at the least be due to a differential inhibition of the fungicide on specific reductase steps. Therefore, our results suggest that reductases of $P$. griseola $f$. mesoamericana that catalyze the conversion of 1,3,6,8 THN to scytalone are more sensible to tricyclazole than those generating vermelone. Kogej et al. (2004) also reported that reductases involved in the different steps of melanin synthesis from both Colletotrichum lagenarium and Magnaporthe grisea showed different sensitivities to tricyclazole.

The effect of tricyclazole on fungal growth and pigmentation also was accompanied with alterations in morphology of black fungi, more precisely the hyphal wall, at the ultrastructural level (Franzen et al. 2006; Kogej et al. 2006). Chattopadhyay et al. (2013) and Kumar et al. (2015) found that the tricyclazole in the range between 25 and $100 \mathrm{ppm}$ provoked several changes in Bipolaris sorokiniana, such as colony morphology, spore size and number of septa in conidia. Also a decrease in the deposition of electron-dense granules in hypha walls was found in cells of Trimmatostroma salinum and T. abietis, chlamydospores of Thielaviopsis basicola, and sclerotia of Botrytis cinerea and Sclerotinia sclerotiorum, when they were cultured in a medium with 1-30 ppm of tricyclazole (Wheeler and Stipanovic 1979; van de Sande et al. 2007; Butler et al. 2009). Our observations show that the hyphal walls of the isolate T4 had an inner electron-transparent layer and an outer electron-dense one, which also has been described for walls of spores from Cladosporium cladosporioides (Latgé et al. 1988) and Humicola grisea (Ellis and Griffiths 1974). Tricyclazole at $100 \mathrm{ppm}$ reduced stratification and deposition of melanin in the hyphal wall of isolate T4, as seen by TEM and CM respectively. Kumar et al. (2015) also associated the presence
Fig. 8 Relation of fluorescence signal for averaging area in cell wall and intracellular vesicles in control cultures $(0)$ and cultures supplemented with tricyclazole (100) treated with $\mathrm{H}_{2} \mathrm{O}_{2}$-light (black bars) and non-treated with $\mathrm{H}_{2} \mathrm{O}_{2}$-light (white bars). Bars with the same capital letter are not significantly different (Student's $t$ Test, $p<0.05)$

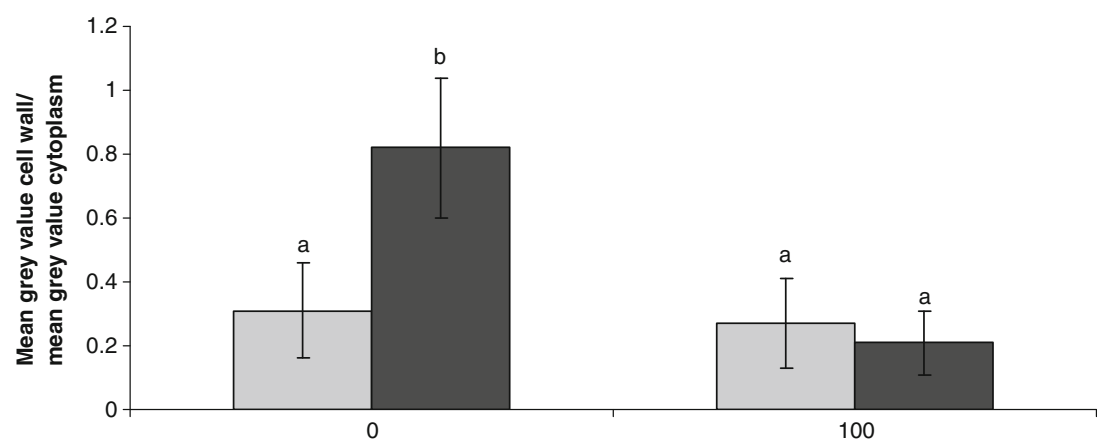


Fig. 9 Growth (a) and pigmentation (b) of colonies of P.griseola mesoamericana isolate T4 on PDA in the absence $(0)$ and presence $(12.5,25,100$ and $200 \mathrm{ppm}$ ) of tricyclazole for a 21 day-period. Values are means of three replicates. Bars with the same capital letter are not significantly different $(p<0.05)$. This experiment was performed twice with similar results
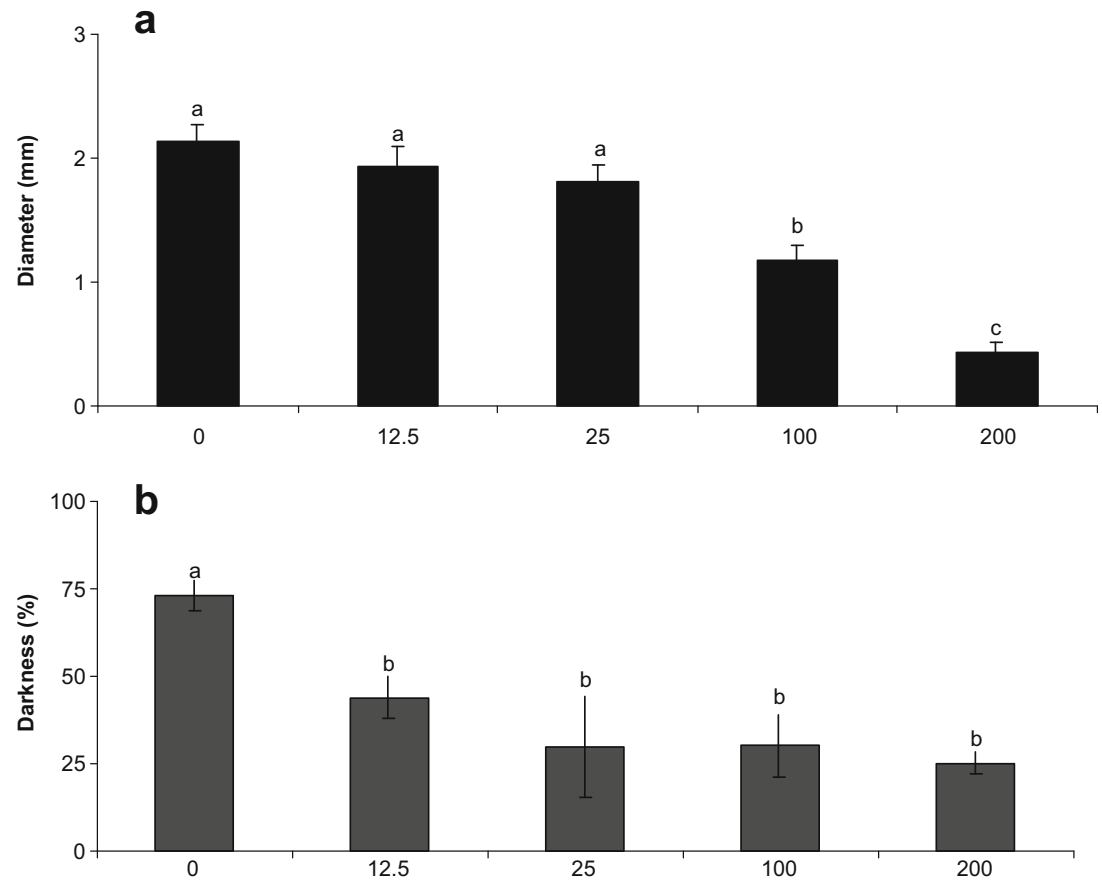

of this inhibitor with morphological changes in the cell walls of hyphae and conidia of the black $B$. sorokiniana isolates, which also was associated with a lower level of melanization. The organization of the hyphal wall in phytopathogens and the localization and amount of their dark pigments has been reported to be an important factor in protecting of several dark fungi from various environmental insults (Mandal et al. 2007). Therefore, tricyclazole might provide an efficient method of control of such phytopathogenic fungi.

The bases of activity of tricyclazole in the pathogen-plant interaction reside on its ability to alter several mechanisms, such as those related to fungal biology as well as the hormonal and antioxidant systems of plants (Habibzade et al. 2012; Shanmugapriya et al. 2013). Tricyclazole has been used to control of $M$. grisea on rice. Recently, it has been demonstrated that this molecule also has fungicide activity against other pathogens of cereals, such as Bipolaris oryzae and B. sorokiniana, Blumeria graminis and Rhynchosporium secalis, as well as Colletotrichum lagenarium and C. lindemuthianum, pathogens of cucumber and beans, respectively (Mares et al. 2004; Gupta et al. 2013; Kumar and Mukherjee 2013; Kumar et al. 2015). Although Saparrat et al. (2009) reported the effect of tricyclazole in melanization of Pseudocercospora griseola, there is no additional data on the role this compound might play in the control of ALS. However, all the evidences presented in this work suggest that tricyclazole might be an effective fungicide for ALS control.

Tricyclazole affected Pseudocercospora griseola $f$. mesoamericana isolate $\mathrm{T} 4$, in several different ways. The most evident effect was on pigment synthesis. It reduced melanin synthesis, and therefore diffusible shunt product from an intermediary of melanin synthesis, identified by UV-Maldi MS, was found. In addition to this, we also found that tricyclazole affected the cell wall structure of mycelia, revealed macroscopically by the reduction in growth. As suggested by Kunova et al. (2012), the knowledge about tricyclazole's mode of action that controls pathogenic fungi is crucial information to support its registration. However, information regarding the efficiency of tricyclazole in field experiments is not available. Future studies should be aimed to evaluate if this molecule can be used to control fungal diseases provoked by DHN-melanized fungi in the field. So far, Pseudocercospora griseola has not been controlled by inhibiting secondary metabolites like melanin that play a biological role. Therefore, the question arises about the effect that an inhibitor of pigment synthesis might have on the pathogenic activity and/or virulence of the fungus. To address this question, future work should be aimed at generating fungal mutants impaired in pigment synthesis.

Acknowledgments We thank Mirífico, M. V. and Rozas, M. F. from the Instituto de Investigaciones Fisicoquímicas teóricas y aplicadas (INIFTA), La Plata (Argentina) for their assistance in performing the FTIR analysis. Bárcena, A. is recipient of a scholarship from the CONICET, Argentina. Erra-Balsells, R., Estévez, J. M., Petroselli, G. and Saparrat, M. C. N. are researchers from the CONICET, Argentina. Balatti, P. A. is a researcher from the CICPBA, Argentina. This work was supported by the Agencia Nacional, PICT2012-2760 to Balatti, P.; PICT2013-003 to Estévez, J. M., PICT 2012-0888 to Erra-Balsells, R., CONICET (PIP 112201101 00391; PIP 0072CO), UBA (0055BA), CICPBA and UNLP, Argentina. 


\section{References}

Bell AA, Wheeler MH (1986) Biosynthesis and functions of fungal melanins. Annu Rev Phytopathol 24:411-451

Beltrán-García MJ, Prado FM, Oliveira MS, Ortiz-Mendoza D, Scalfo AC, Pessoa A Jr, Medeiros MHG, White JF, Di Mascio P (2014) Singlet molecular oxygen generation by light-activated DHN-melanin of the fungal pathogen Mycosphaerella fijiensis in Black Sigatoka Disease of Bananas. PloS one 9, e91616. doi:10.1371/ journal.pone.0091616

Butler MJ, Day AW (1998) Fungal melanins: a review. Can J Microbiol 44:1115-1136

Butler MJ, Gardiner RB, Day AW (2009) Melanin synthesis by Sclerotinia sclerotiorum. Mycologia 101:296-304

Carzaniga R, Fiocco D, Bowyer P, O'Connell R (2002) Localization of melanin in conidia of Alternaria alternata using phage display antibodies. Mol Plant Microbe Interact 15:216-224

Chaskes S, Frases S, Cammer M, Gerfen G, Casadevall A (2008) Growth and pigment production on D-tryptophan medium by Cryptococcus gattii, Cryptococcus neoformans, and Candida albicans. J Clin Microbiol 46:255-264

Chattopadhyay A, Kushwaha C, Chand R, Srivastava JS (2013) Differential mode of action of tricyclazole in vitro and in planta on Bipolaris sorokiniana causing spot blotch in barley. Indian Phytopathol 66:155-158

Cooke RC, Whipps JM (1993) Ecophysiology of fungi. Blackwell Scientific Publications, London

Crous PW, Liebenberg MM, Braun U, Groenewald JZ (2006) Reevaluating the taxonomic status of Phaeoisariopsis griseola, the causal agent of angular leaf spot of bean. Stud Mycol 55:163-173

Ddamulira G, Mukankusi C, Ochwo-Ssemakula M, Edema R, Sseruwagi P, Gepts P (2014) Distribution and variability of Pseudocercospora griseola in Uganda. J Agric Sci 6:16-29

Elliott ML (1995) Effect of melanin biosynthesis inhibiting compounds on Gaeumannomyces species. Mycologia 87:370-374

Ellis DH, Griffiths DA (1974) The location and analysis of melanins in the cell walls of some soil fungi. Can J Microbiol 20:1379-1386

Fernandez CW, Koide RT, Newsham K (2013) The function of melanin in the ectomycorrhizal fungus Cenococcum geophilum under water stress. Fungal Ecol 6:479-486

Franzen AJ, Cunha MML, Batista EJO, Seabra SH, Souza WDE, Rozental S (2006) Effects of Tricyclazole (5-methyl-1,2,4triazol[3,4] benzothiazole), a specific DHN-melanin inhibitor, on the morphology of Fonsecaea pedrosoi conidia and sclerotic cells. Microsc Res Tech 69:729-737

Franzen AJ, Cunha MML, Miranda K, Hentschel J, Plattner H, da Silva MB, Salgado CG, de Souza W, Rozental S (2008) Ultrastructural characterization of melanosomes of the human pathogenic fungus Fonsecaea pedrosoi. J Struct Biol 162:75-84

Griffith GW, Easton GL, Detheridge A, Roderick K, Edwards A, Worgan HJ, Nicholson J, Perkins WT (2007) Copper deficiency in potato dextrose agar causes reduced pigmentation in cultures of various fungi. FEMS Microbiol Lett 276:165-171

Gupta V, Naveed S, Razdan VK, Sharma BC, Rishu S, Kavaljeet K, Ichpal S, Dolly J, Kumar A (2013) Foliar application of fungicides for the management of brown spot disease in rice (Oryza sativa L.) caused by Bipolaris oryzae. Afr J Agric Res 8:3303-3309

Habibzade F, Sorooshzadeh A, Pirdashti H, Mohammad SA (2012) Effect of nitrogen compounds and tricyclazole on some biochemical and morphological characteristics of waterlogged-canola. Int Res J Appl Basic Sci 3:77-84

Hoss R, Helbig J, Bochow H (2000) Function of host and fungal metabolites in resistance response of banana and plantain in the black sigatoka disease pathosystem (Musa spp -Mycosphaerella). J Phytopathol 148:387-394
Kayatz P, Thumann G, Luther TT, Jordan JF, Bartz-Schmidt KU, Esser PJ, Schraermeyer U (2001) Oxidation causes melanin fluorescence. Invest Ophthalmol Vis Sci 42:241-246

Kogej T, Wheeler MH, Lanisnik RT, Gunde-Cimerman N (2004) Evidence for 1,8-dihydroxynaphthalene melanin in three halophilic black yeasts grown under saline and non-saline conditions. FEMS Microbiol Lett 232:203-209

Kogej T, Gorbushina AA, Gunde-cimerman N, Money NP (2006) Hypersaline conditions induce changes in cell-wall melanization and colony structure in a halophilic and a xerophilic black yeast species of the genus Trimmatostroma. Mycol Res 110:713-724

Kumar N, Mukherjee I (2013) Effect of soil physicochemical properties on adsorption of Tricyclazole. Int J Agric Food Sci Technol 4:391396

Kumar M, Chand R, Dubey RS, Shah K (2015) Effect of Tricyclazole on morphology, virulence and enzymatic alterations in pathogenic fungi Bipolaris sorokiniana for management of spot blotch disease in barley. World J Microbiol Biotechnol 31:23-35

Kunova A, Pizzatti C, Cortesi P (2012) Impact of tricyclazole and azoxystrobin on growth, sporulation and secondary infection of the rice blast fungus, Magnaporthe oryzae. Pest Manag Sci 69: $278-284$

Langfelder K, Streibel M, Jahn B, Haase G, Brakhage AA (2003) Biosynthesis of fungal melanins and their importance for human pathogenic fungi. Fungal Genet Biol 38:143-158

Latgé JP, Bouziane H, Diaquin M (1988) Ultrastructure and composition of the conidial wall of Cladosporium cladosporioides. Can J Microbiol 34:1325-1329

Lazarovits G, Stoessl A (1988) Tricyclazole induces melanin shunt products and inhibits altersolanol a accumulation by Alternaria solani. Pestic Biochem Physiol 31:36-45

Llorente C, Bárcena A, Vera Bahima J, Saparrat MCN, Arambarri AM, Rozas MF, Mirífico MV, Balatti PA (2012) Cladosporium cladosporioides LPSC 1088 produces the 1,8 dihydroxynaphthalene-melanin-like compound and carries a putative pks gene. Mycopathologia 174:397-408

Mandal P, Roy TS, Das TK, Banerjee U, Xess I, Nosanchuk JD (2007) Differences in the cell wall architecture of melanin lacking and melanin producing Cryptococcus neoformans clinical isolates from India: an electron microscopic study. Braz J Microbiol 38:662-666

Mares D, Romagnoli CR, Andreotti E, Manfrini MM, Vicentini CB (2004) Synthesis and antifungal action of new Tricyclazole analogues. J Agric Food Chem 52:2003-2009

McLafferty FW, Turecek F (1993) Interpretation of Mass Spectra. University Science Books, Mill Valley

Moriwaki A, Kihara J, Kobayashi T, Tokunaga T, Arase S, Honda Y (2004) Insertional mutagenesis and characterization of a polyketide synthase gene (PKS1) required for melanin biosynthesis in Bipolaris oryzae. FEMS Microbiol Lett 238:1-8

Nobre MO, Ferreiro L (2004) Production and evaluation of albino mutants of Sporothrix schenckii. Acta Sci Vet 32:119-123

Nonami H, Fukui S, Erra-Balsells R (1997) $\beta$-Carboline alkaloids as matrices for matrix-assisted ultraviolet laser desorption time-of flight mass spectrometry of proteins and sulfated oligosaccharides: a comparative study using phenylcarbonyl compounds, carbazoles and classical matrices. J Mass Spectrom 32:287-296

Onofri S, Barreca D, Selbmann L, Isola D, Rabbow E, Horneck G (2008) Resistance of antarctic black fungi and cryptoendolithic communities to simulated space and Martian conditions. Stud Mycol 61:99 109

Paparu P, Katafiire M, Mcharo M, Ugen M (2014) Evaluation of fungicide application rates, spray schedules and alternative management options for rust and angular leaf spot of snap beans in Uganda. Int $\mathrm{J}$ Pest Manag 60:82-89

Ricardi M, Guaimas FF, González RM, Burrieza HP, López-Fernández MP, Jares-Erijman EA, Estévez JM, Iusem ND (2012) Nuclear 
import and dimerization of tomato ASR1, a water stress-inducible protein exclusive to plants. PloS one 7, e41008. doi:10.1371/journal. pone. 0041008

Romero-Martinez R, Wheeler M, Guerrero-Plata A, Rico G, TorresGuerrero H (2000) Biosynthesis and functions of melanin in Sporothrix schenckii. Infect Immun 68:3696-3703

Saparrat MCN, Fermoselle G, Stenglein S, Aulicino M, Balatti PA (2009) Pseudocercospora griseola causing angular leaf spot on Phaseolus vulgaris produces 1,8-dihydroxynaphthalene-melanin. Mycopathologia 1:41-47

Saparrat MCN, Jurado M, Díaz R, Romera IG, Martínez MJ (2010) Transformation of the water soluble fraction from "alpeorujo" by Coriolopsis rigida: the role of laccase in the process and its impact on Azospirillum brasiliense survival. Chemosphere 78:72-76

Schwartz HF, Galvez GE (1980) Problemas de producción del frijol: Enfermedades, insectos, limitaciones edáficas y climáticas de Phaseolus vulgaris. Centro Internacional de Agricultura Tropical (CIAT), Cali

Shanmugapriya AK, Sivakumar T, Panneerselvam R (2013) Difenoconazole and Tricyclazole induced changes in photosynthetic pigments of Lycopersicon esculentum. L. Int J Agric Food Sci 3:7275

Singaravelan N, Grishkan I, Beharav A, Wakamatsu K, Ito S, Nevo E (2008) Adaptive melanin response of the soil fungus Aspergillus niger to UV radiation stress at "Evolution Canyon", Mount Carmel, Israel. PloS one 3, e2993. doi:10.1371/journal.pone.0002993

Stenglein SA, Balatti PA (2006) Genetic diversity of Phaeoisariopsis griseola in Argentina as revealed by pathogenic and molecular markers. Physiol Mol Plant Pathol 68:158-167

Stenglein SA, Ploper D, Vizgarra O, Balatti PA (2003) Angular leaf spot: a disease caused by the fungus Phaeoisariopsis griseola (Sacc.) Ferraris on Phaseolus vulgaris L. In: Laskin AI, Bennett JW,
Gadd GM (eds) Advances in Applied Microbiology, vol 52. Academic Press, San Diego, pp 209-243

Suryanarayanan TS, Ravishankar JP, Venkatesan G, Murali TS (2004) Characterization of the melanin pigment of a cosmopolitan fungal endophyte. Mycol Res 108:974-978

Taborda CP, Silva MB, Nosanchuk JD, Travassos LR (2008) Melanin as a virulence factor of Paracoccidioides brasiliensis and other dimorphic pathogenic fungi: a minireview. Mycopathologia 165:331-339

Tavzes C, Silc F, Kladnik A, Fackler K, Messner K (2009) Enzymatic degradation of mould stains on paper analysed by colorimetry and DRIFT-IR spectroscopy. Int Biodeterior Biodegrad 63:873-879

Tu Y, Sun Y, Tian Y, Xie M, Chen J (2009) Physicochemical characterisation and antioxidant activity of melanin from the muscles of Taihe Black-bone silky fowl (Gallus gallus domesticus Brisson). Food Chem 114:1345-1350

van de Sande WWJ, de Kat J, Coppens J, Ahmed AOA, Fahal A, Verbrugh H, van Belkum A (2007) Melanin biosynthesis in Madurella mycetomatis and its effect on susceptibility to itraconazole and ketoconazole. Microbiol Infect 9:1114-1123

Vizgarra ON, Balatti PA, Stenglein SA, Espeche CM, Ploper LD (2010) TUC 550: nueva variedad de poroto negro (Phaseolus vulgaris L.) con tolerancia a la mancha angular (Phaeoisariopsis griseola). Rev Ind Agríc Tucumán 87:55-61

Wheeler MH, Klich MA (1995) The effect of tricyclazole, pyroquilon, phthalide, and related fungicides on the production of conidial wall pigments by Penicillium and Aspergillus species. Pestic Biochem Physiol 52:125-136

Wheeler MH, Stipanovic RD (1979) Melanin Biosynthesis in Thielaviopsis basicola. Exp Mycol 3:340-350

Zeng J, Decker R, Zhan J (2012) Biochemical characterization of a type III polyketide biosynthetic gene cluster from Streptomyces toxytricini. Appl Biochem Biotechnol 166:1020-1033 